\title{
Q5 \\ Drug discovery through stem cell-based organoid models
}

\section{Q3 Q1 Adrian Ranga, Nikolche Gjorevski, Matthias P. Lutolf}

Laboratory of Stem Cell Bioengineering, Institute of Bioengineering, Ecole Polytechnique Fédérale de Lausanne (EPFL), Switzerland

\section{A R T I C L E I N F O}

\section{Article history:}

Accepted 18 February 2014

Available online $\mathrm{xxxx}$

\section{Contents}

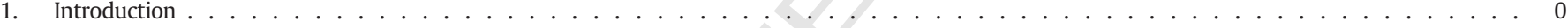

2. From phenotype to organotype: high-throughput screening and the 3D paradigm . . . . . . . . . . . . . . . . . . . . 0

2.1. Phenotypic screens: an additional dimension . . . . . . . . . . . . . . . . . . . . . . . . . . . 0

2.2. Scaffold-free multicellular cancer tumor spheroids . . . . . . . . . . . . . . . . . . . . . . . . . . . . 0

2.3. Scaffold-based multicellular cancer tumor spheroids . . . . . . . . . . . . . . . . . . . . . . . . 0

2.4. Migration, invasion and angiogenesis in $3 \mathrm{D} \ldots \ldots \ldots \ldots \ldots$

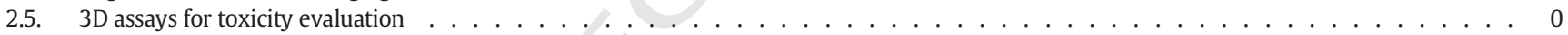

3. Organoids as in vitro organ models: promise and challenges . . . . . . . . . . . . . . . . . . . . . . . . . . . . . . . . . 0

4. State-of-the-art organoid culture systems: Matrigel ${ }^{\mathrm{TM}}$ as critical component . . . . . . . . . . . . . . . . . . . . . . . . . . . . . . 0

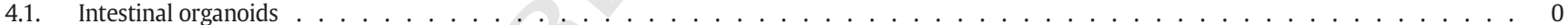

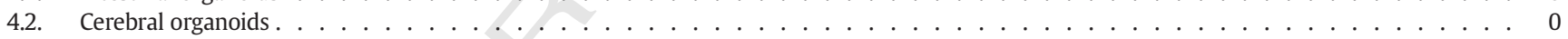

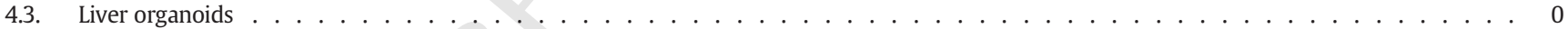

5. ECMs and 3D screening: towards synthetics and scalable approaches . . . . . . . . . . . . . . . . . . . . . . . . . . . . . . 0

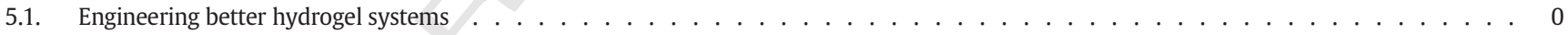

5.2. High-throughput approaches for microenvironment optimization . . . . . . . . . . . . . . . . . . . . . . . . . . 0

5.3. Challenges in implementing engineered organoids . . . . . . . . . . . . . . . . . . . . . . . . . 0

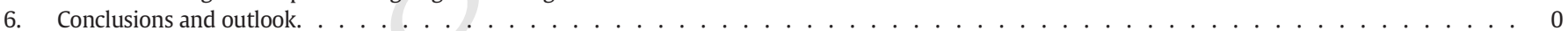

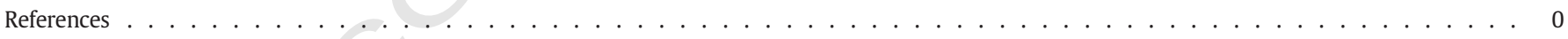

\section{Q7 Q6 1. Introduction}

The development of new drugs is currently a long and costly process in large part due to the failure of promising drug candidates identified in initial in vitro screens to perform as intended in vivo. New approaches to drug screening are being developed which focus on providing more biomimetic platforms. This review surveys this new generation of drug screening technologies, and provides an overview of recent

\footnotetext{
is This review is part of the Advanced Drug Delivery Reviews theme issue on "Innovative tissue models for in vitro drug development".

E-mail addresses: adrian.ranga@epfl.ch (A. Ranga), matthias.lutolf@epfl.ch (M.P. Lutolf).
}

developments in organoid culture systems which could afford previous- 44 ly unmatched fidelity for testing bioactivity and toxicity. The challenges 45 inherent in such approaches will also be discussed, with a view towards 46 bridging the gap between proof-of-concept studies and a wider imple- 47 mentation within the drug development community.

Drug discovery today is at a crossroads: while increasingly large and 49 varied compound libraries are synthesized and tested in primary 50 screens, the promise of the identified lead compounds remains largely 51 unrealized. Indeed, while tremendous investments in automation 52 have enabled the costs and turnaround time for large to medium-scale 53 primary screening to fall significantly [1], the gap between lead com- 54 pound validation and success in the clinic is still wide, suggesting that 55 a process still beset by significant limitations in efficiency. 
To a significant degree, this inefficiency in taking lead compounds into the clinic may be due to the discrepancy between the simplified in vitro assays currently performed and the complexity of real in vivo pathologies. Indeed, while both drug safety and efficacy intrinsically linked to administration into a complex and heterogeneous three-dimensional (3D) physiological system, most primary drug screening campaigns are still carried out with cell lines grown on two-dimensional (2D) plastic, an entirely reductionist approach where important parts of the drugbiology interaction are lost. The outcome of this primary screening process is the identification of "hits", which satisfy very specific molecular targets or phenotypic requirements. A key problem is that these lead compounds are then validated and optimized in similarly oversimplified culture models. The process of ADMET evaluation (adsorption, distribution, metabolism, excretion, toxicology), while having undergone significant improvements in the last 15 years [2], could still be considered one of the main bottlenecks in the drug development process and could afford the greatest return on technological innovations [3].

As an important additional requirement, regulatory agencies require that identified pro-drugs be tested in two animal models before granting approval to proceed to any human clinical trials. This costly process of validation in animal models often fails due to physiological events linked to fundamental differences between human and animal model physiology. At this increasingly costly step, due to well-known differences in mechanisms of metabolism and toxicology between species, there remains a significant lack of fidelity between current testing procedures and human outcomes, particularly as related to appropriate evaluation of toxicity and drug dose.

These shortcomings have been clearly recognized within the pharmaceutical industry [4], yet few fundamental solutions have currently been implemented. The behavior of cells and their response to drugs continue to be studied in vitro mostly in 2D cell cultures that completely fail to mimic the complexity of the microenvironment. Not surprisingly, drug responsiveness in these settings is therefore often not predictive of the in vivo situation, which dramatically increases the costs of drug 91 discovery.

At the same time, a vast amount of research has been carried out in 93 academia to develop more relevant test-beds for screening and valida- 94 tion efforts (Fig. 1). In particular, there has been a push towards the 95 development of multicellular spheroid models [5], notably in cancer 96 modeling [6], as well as a number of miniaturized approaches culminat- 97 ing in organ-on-chip systems [7]. More recently, there has been a tre- 98 mendous interest in developing increasingly complex multicellular 99 constructs termed "organoids" [8-10] (Fig. 2). These morphogenetic 100 models, often recapitulating developmental programs from embryology 101 or harnessing adult stem cell-based regenerative processes, have 102 allowed molecular and cell biologists to understand key signaling 103 events required for the initiation and maintenance of multicellular 104 organs. By recapitulating not only the form but also the rudiments of $\mathbf{Q 8}$ function of their in vivo counterparts, these constructs have the poten- 106 tial to move from laboratory proof-of-concepts to relevant tools in the 107 drug discovery pipeline. Indeed, such organoids could finally provide a 108 key missing link between compound screening and clinical trials, and 109 could serve as models for testing drug efficacy in target organs, for 110 toxicity in liver models or for bioavailability through intestinal system 111 models. In particular, by using primary human cells, especially 112 patient-derived cells with relevant pathologies in conjunction with 113 cellular reprogramming strategies, these techniques could provide an 114 invaluable link to disease-specific human drug screening models. 115

Ultimately, the wider implementation of these bio-mimicking ap- 116 proaches within a still-conservative drug development community 117 will require the level of reproducibility and consistency currently 118 achieved with cell lines. Thus, such culture models will require 3D cul- 119 ture conditions which afford the needed flexibility to achieve precise 120 control over the cellular microenvironment as well as a level of scalabil- 121 ity. Furthermore, the applicability of such models will be greatly en- 122 hanced by adapting to existing infrastructure, notably automatic 123 robotic platforms for experimental setup and assay readouts.

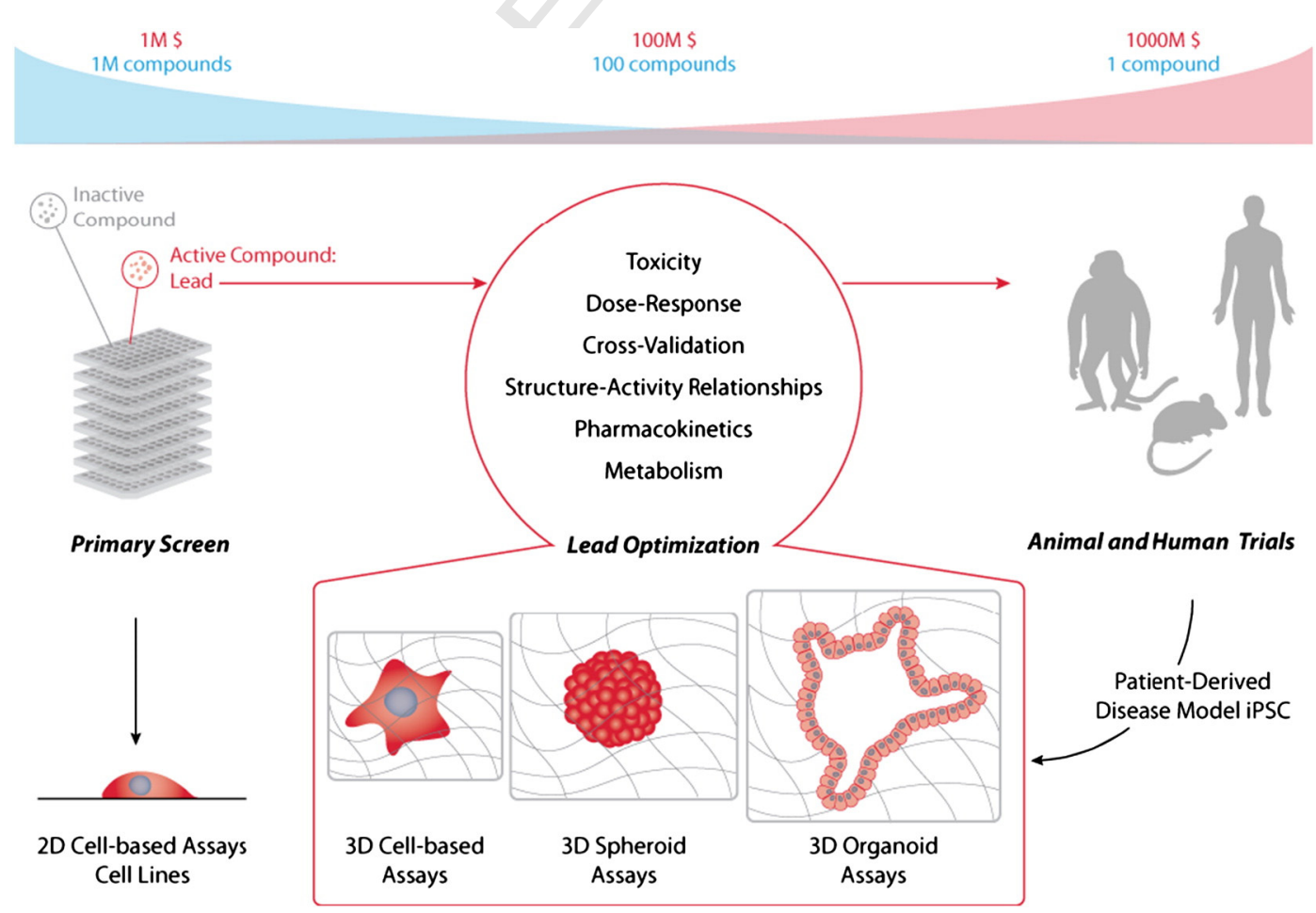

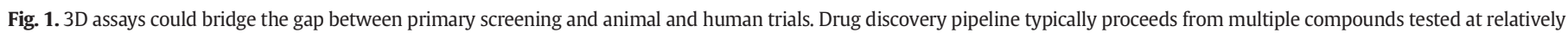
low cost to few compounds in high-cost high-risk trials. The process of lead optimization and validation can benefit from increasingly representative in vitro technologies. 
A

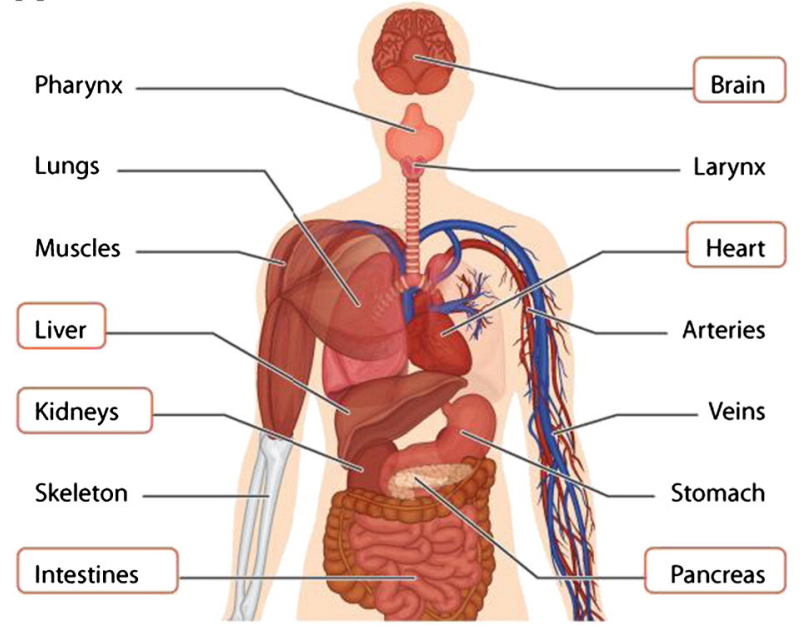

B
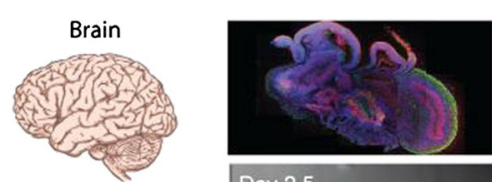

Intestine

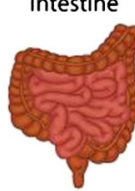

Liver

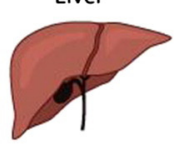

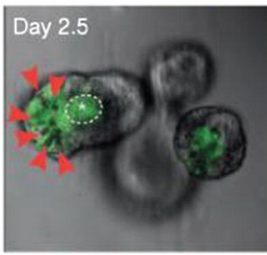

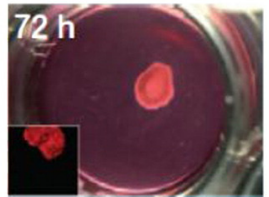

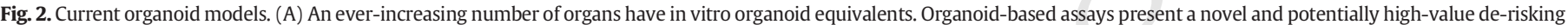

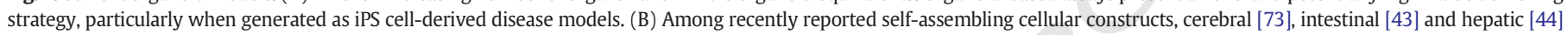
organoids are prominent examples of organoid cultures with potential applications in drug discovery.

Reproduced by permission of Nature Publishing Group.

Thus the purpose of this review is first to provide a selected survey of existing state-of-the-art 3D models of in vitro drug evaluation, then to introduce some key recent developments in organoid systems, and finally, through a critical evaluation of the limitations of such systems, to propose some advances which could lead to the adoption of such models by making the case for a real functional value in helping derisk this process.

\section{From phenotype to organotype: high-throughput screening and the 3D paradigm}

\subsection{Phenotypic screens: an additional dimension}

Phenotypic drug discovery has become increasingly popular in early stage drug discovery. Unlike target-based screens, in phenotypic screens, there is no a priori understanding of the molecular mechanism of action, and the effect of compounds on cell phenotype is observed directly. Such an approach has emphasized the importance of biologically-focused assays: between 1999 and 2008, in a period where target-based screens still predominated the drug discovery process, out of 75 first-in-class drugs with new molecular mechanism of action approved by the US Food and Drug Administration, the contribution of phenotypic screening to the discovery of first-in-class small-molecule drugs exceeded that of target-based approaches - with 28 and 17 of these drugs coming from the two approaches, respectively [11]. As cell-based assays continue to gain prominence and widespread adoption, it is clear that the new approaches focused on further enhancing biological relevance are necessary.

In the last few years, there has been a significant effort to develop 3D culture systems which better represent in vivo biology. Within this vast field, there have also been numerous approaches focused on highthroughput and miniaturized implementations of such technologies. Here, we will present such approaches, particularly focusing on most recent developments pertaining to implementation in high-throughput systems. Oncology has been one of the most important targets of drug discovery; in this field where the presentation of the pathology is often heterogeneous, and drug effectiveness, resistance and toxicity manifest itself in many ways. It is therefore in this field that a number of advances in the creation of more physiologically relevant approaches have been most prominent. Indeed, using a number of established cell lines, the cancer spheroid model as well as complementary assays for 162 invasion, migration, and angiogenesis have been most readily explored. 163

\subsection{Scaffold-free multicellular cancer tumor spheroids}

Multicellular cancer tumor spheroids (MCTS) represent a well- 165 established in vitro model for avascular tumor growth and this model 166 has become a classic reference for 3D studies [5,12]. By forming an aggre- 167 gate of cells which form cell-cell interactions, striking changes in mor- 168 phology and gene expression are evidenced [13] (Fig. 3A) as a number 169 of pathophysiological characteristics of an in vivo tumor are recreated, 170 notably, oxygen gradients, glucose distribution, lactate accumulation, 171 DNA strand breaks, ATP distribution and histomorphology/proliferation 172 characteristics [14]. It is also particularly appropriate as numerous cancer 173 cell lines, particularly those from the NCI-60 DTP human tumor cell line 174 screen established by the NIH, have been shown to form spheroids with- 175 out the addition of exogenous matrix materials [14].

For screening purposes, spheroid-forming assays have been devel- 177 oped in a most rigorously standardized manner within liquid media 178 cultures. A key consideration in such studies has been the control over 179 spheroid size, with the intention to establish a spheroid-based screen 180 with clear pathophysiological gradients but without central necroses 181 at the onset of treatment [15]. Indeed, a spheroid size of $400 \mu \mathrm{m}$ was 182 found to be ideal to recreate hypoxic conditions at the core, as well as 183 proliferative gradients, which have significant impact on radio and 184 drug-resistance as well as indirect effects of hypoxia-driven gene ex- 185 pression. Monitoring of spheroid growth kinetics to determine growth 186 delay and regrowth upon drug administration consists of the primary 187 analytical endpoint, and is performed by standard phase-contrast imag- 188 ing. The acid phosphatase assay (APH) has also been established to 189 monitor cell integrity and viability, with IC50 values estimated from 190 dose-response curves determined through such an APH cell viability 191 assessment.

Such a basic approach has been enhanced with the use of specifically 193 designed high-density plates which have been engineered to allow for 194 high-throughput hanging drop culture systems. Indeed such systems 195 have been optimized for droplet stability [16], have been shown to be 196 robust in fluorescence- and colorimetric-based assays through Z-factor 197 calculations [17], and have allowed for the determination of differential 198 effects on growth arrest with drugs. For example, fluoracil (5-FU) was 199 determined to be more effective as an anti-proliferative agent in 2D, 200 
A

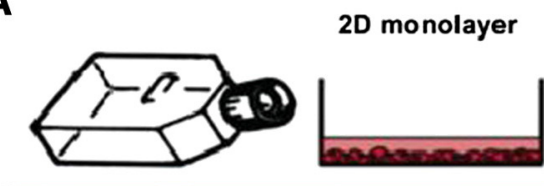

conventional 2D growth on plastic dish

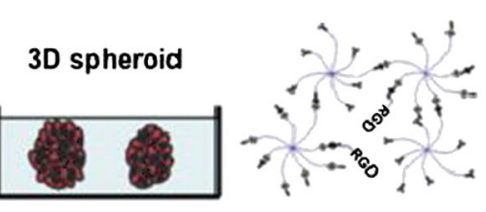

3D embedded growth using PEG hydrogel

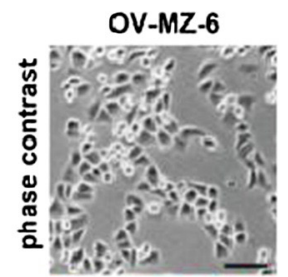

SKOV-3
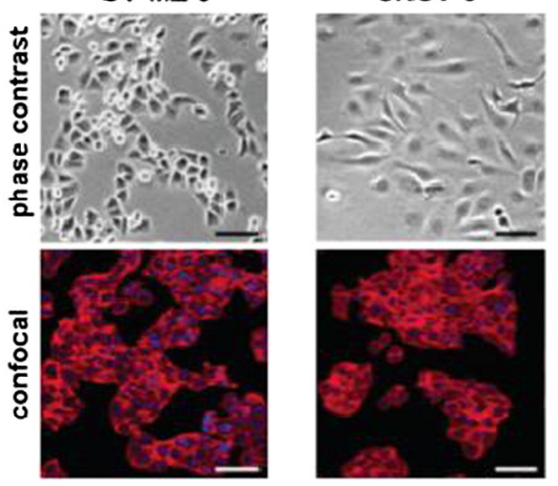

B

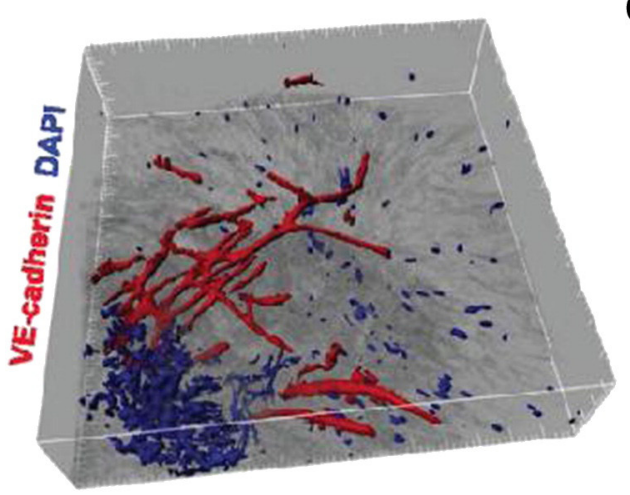

OV-MZ-6

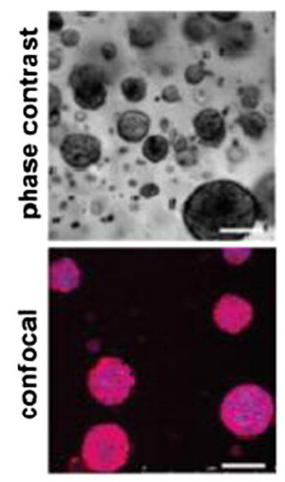

SKOV-3

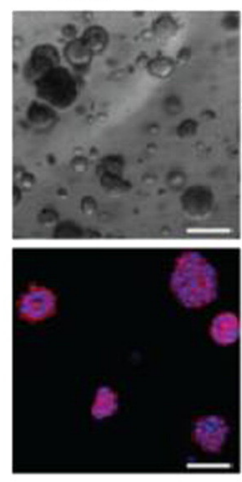

C
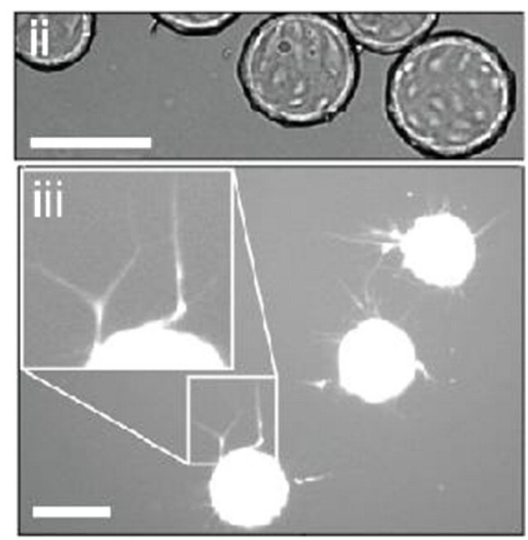

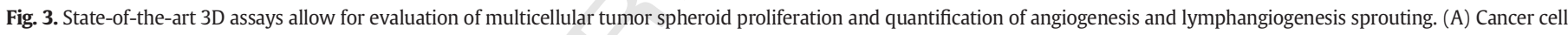

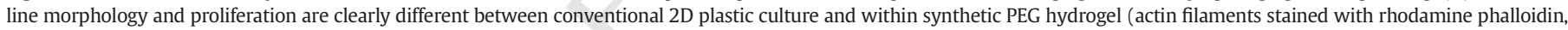

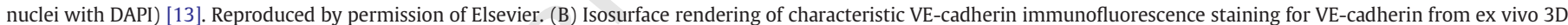

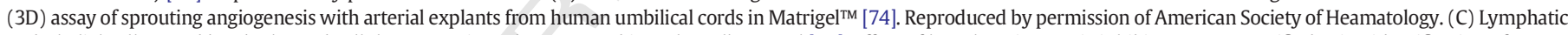

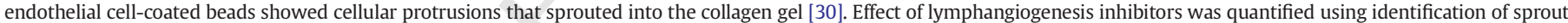
number per bead by in-house developed software. Reproduced by permission of National Academy of Sciences of the United States of America.

whereas hypoxia activated drugs such as tirapazamine (TPZ) were seen to be more effective in 3D hanging drop cultures [18]. Hanging drop culture systems have also been recently used in an elegant highthroughput manner to show how co-culture models of cancer and stromal cells could unveil novel regulatory pathways [19].

\subsection{Scaffold-based multicellular cancer tumor spheroids}

The generation of large-scale liquid media spheroids has been the focus of technological advances in up-scaling; by creating "droplet microarrays" with the possibility of generating thousands of droplets with defined geometry and volume using superhydrophilic-superhydrophobic patterned surfaces [20], or enhancements in hanging drop spheroid manipulation through incorporation of magnetic-based technologies [21].

While these techniques utilizing hanging drop or liquid suspension cultures provide an undeniable ease of use, such a free-growing construct certainly does not recapitulate the physical constrains experienced by in vitro tumors and may miss important mechanisms of extracellular interaction-mediated drug resistance. In order to address this issue, multiple platforms have been developed to allow cancer 219 spheroids to be grown within simplified extracellular matrices (ECM). 220 To cite only a few recent examples, methylcellulose has been used as a 221 simple 3D culture system for pancreatic ductal carcinoma cells and 222 compared to standard 2D culture conditions; in the 3D case cell metab- 223 olism was seen to shift towards glycolysis, and, notably, while most 224 drugs tested were shown to be less effective in 3D, two were identified 225 as having particularly significant effects in this model [22]. In another 226 study a 3D soft agar matrix was adapted to high-throughput screening, 227 and 1528 natural product compounds were screened against colorectal 228 carcinoma colonies [23]. Notably, this study showed how by comparing 229 tumor-only cells with a co-culture model incorporating colon epithelial 230 cells, it was possible to distinguish tumor-specific agents from general 231 cytotoxic ones. A number of these technologies are now commercially 232 available and have been shown to be compatible with a number of 233 downstream assays beyond imaging. For example, in alginate-based 234 scaffolds while cytotoxicity was measured by AlamarBlue ${ }^{\circledR}$ assay and 235 drug effectiveness was measured by imaging, additional readouts such 236 as apoptosis were evaluated by immunohistochemistry and RT-PCR, 237 where cellular uptake of drugs and nanoparticles could also be 238 
evaluated [24]. Again, it was shown that IC50 values for a number of cancer drugs were significantly higher in a spheroid model as compared to 2D. Thus, as demonstrated in these selected examples, the multicellular tumor spheroid model, despite its simplicity as a first approximation of a 3D tumor microenvironment, has already shown value in a high-throughput drug discovery pipeline.

\subsection{Migration, invasion and angiogenesis in $3 D$}

While aberrant cellular proliferation may be the primary manifestation of solid tumor cancers, it is via remodeling and migration through the tissue microenvironment that malignant cells metastasize to spread to adjacent tissues or distant sites via lymphatic or angiogenic means. A better understanding of the role of the microenvironment in initiating and promoting these processes is critical if the prevention of metastasis is to be used as a target for oncologic therapeutics. With appropriate 3D culture models, it is possible to monitor not only bulk volume increases of spheroids (i.e. tumor proliferation), but also, given appropriate matrix conditions and chemotactic cues, the outgrowth of individual cells from such spheroids (i.e. cell migration). A number of approaches to assay migration and invasion exist, with varying degrees of physiological relevance and ease of implementation [25]. A prominent example includes filter-based transwell assays, which can be additionally coated with a layer of ECM components such as collagen or Matrigel ${ }^{\mathrm{TM}}$. More complex models incorporate a level of dimensionality, such as "sandwich assays" where a monolayer of cells is entrapped between two layers of ECM. Most relevantly, cellular spheroids such as the ones described above can be seeded onto a relevant matrix, or completely embedded within a 3D matrix [25]. Angiogenesis is one of the central hallmarks of cancer progression, and significant efforts have been carried out to determine ways to understand and inhibit this process in increasingly biorelevant model systems [26-28] (Fig. 3B). For example, a 3D vascular network assay showed considerable sensitivity to several angiogenic inhibitors, including kinase inhibitors and monoclonal antibodies and led the development of a 3D model of tumor-driven angiogenesis, in which angiogenic outgrowth was sustained by spheroids of prostate cancer cells in the absence of exogenous growth factors [29]. Another notable use of 3D-based spheroid assays was developed to screen for inhibitors of lymphangiogenesis [30], a process akin to angiogenesis and highly implicated in tumor progression (Fig. 3C). In this study, spheroids were formed by coating lymphatic endothelial cells around cytodextran microcarrier beads, and were then tested in a 3D high-throughput sprouting assay over the LOPAC collection of pharmacological compounds. By enhancing the power of such a screen by deploying automated microscopy in conjunction with custom-developed advanced image processing software, novel regulators of lymphangiogenesis could be detected. In particular, a previously unknown link was established between statins and the inhibition of lymphangiogenesis, which has potential implications not only directly in the treatment of cancer but also for interactions with and management of cardiovascular disease.

\subsection{D assays for toxicity evaluation}

While cancer is a primary target for the deployment of advanced high-throughput screening strategies, and has been seen in examples above to benefit from a 3D approach, it is by no means the only area where spheroid-based assays have been used. Indeed the spheroid assay has found significant use in organ- and cell-type-specific toxicology studies, constituting another important step in the drug validation process. While dose response and toxicity must clearly be evaluated in the cell type of interest, systemic toxicity, and particularly liver toxicity, is also of primary concern. As such, toxicity studies are routinely performed in HepG2 cells, a hepatocarcinoma cell line which has been frequently used as a model system to study liver metabolism and cytotoxicity. 3D spheroids of HepG2 and closely related HepaRG ${ }^{\mathrm{TM}}$ cells have been cultured in hanging drop suspension cultures and compared to 2D 301 cultures. In one study, it has been shown that activity of CYP4A4, a 302 member of the P-450 monooxygenases involved in the metabolism of 303 a broad range of compounds from steroids to drugs and toxins, was 304 higher in the 3D cultures compared to 2D [31]. Moreover, 3D cell 305 cultures were more sensitive to a drug that is only toxic upon metabolic 306 activation in the liver (aflatoxin B), suggesting that such an organotypic 307 system better represents in vivo liver metabolism. Notably, within such 308 an organotypic culture, the EC50 of acetaminophen was similar to 309 in vivo toxicity, a phenomenon which could not be reproduced in 2D, 310 demonstrating once again the importance of a 3D model for capturing 311 in vivo response [32]. In a further advance, liver microtissues have 312 also recently been constructed from primary human hepatocytes and 313 liver-derived non-parenchymal cells [33]. Interestingly, significant 314 species-specific differences in drug hepatotoxic response were found 315 between rat and human microtissues [34], highlighting the importance 316 of developing human cell-based 3D culture systems.

\section{Organoids as in vitro organ models: promise and challenges}

Despite the increasingly acknowledged value of in vitro 3D culture, 319 of which some examples were reviewed in the previous section, animal 320 models have remained as the immediate next test bed for promising 321 new compounds after an initial primary 2D screen. However there is 322 a significant gap between the still highly simplified models of spher- 323 oids and the systemic effects seen in an animal, with all the possible 324 confounding effects which cannot be clearly deconvolved. Moreover, 325 there have also been some significant data over the years indicating 326 substantial differences between animal and human modes of drug 327 response. For example, in a survey of a dozen pharmaceutical com- 328 panies, with data from 150 compounds with 221 human toxicity 329 events, data from rodents failed to predict $57 \%$ of incidents of 330 human toxicity [35]. Furthermore, a recent study showed a complete 331 lack of correlation in the genomic response to acute inflammatory 332 stress between human subjects and murine models, suggesting that 333 the use of such unrepresentative models may have accounted, thus 334 far, for the failure of all compounds which have been put through Q9 clinical trials intended to block the inflammatory response in criti- 336 cally ill patients [36].

An ideal in vitro analysis system would therefore comprise of human 338 cells, in a construct complex enough to demonstrate physiologic-like 339 composition, morphology and heterogeneity and, ideally, the rudiments 340 of functionality, yet simple enough that it could still be readily assayed 341 in vitro. Such a construct, capturing some of the complexity of a 342 human organ in a dish, has been termed "organoid" [37] (Fig. 2). The 343 idea of creating in vitro organoids is not a new phenomenon: leveraging 344 cells' intrinsic ability to self-assemble into organized structures has been 345 envisaged at least since the early generation of teratocarcinomas in 346 1954 [38]. Indeed, when embryonal carcinoma cells were transplanted 347 into a host mouse, malignant tumors were found to develop and could 348 form tissues of all germ layers, and surprisingly, could even in rare 349 cases develop into complete organs. This became even more relevant 350 with the advent of embryonic stem cells (ESC), which, under the same 351 condition, i.e. reimplantation into a host animal, could generate equally 352 histologically and morphologically complex structures. These early 353 studies clearly demonstrated the potential of stem cells grown in vitro 354 to recreate complex and organized structures, albeit when placed in 355 the context of a complex host microenvironment. As well, these studies 356 early on demonstrated the need for extrinsic microenvironmental regu- 357 lation for growth and development. In the context of more relevant 358 physiological studies in vitro, clearly there was an interest and a need 359 to understand how such processes could be regulated, in order to then 360 attempt to recreate these processes in vitro.

While the field of tissue engineering has made significant attempts to 362 recreate in vitro organs in the last 30 years, these approaches have gen- 363 erally focused on scaffold-based cell seeding techniques, and arguably 364 
have found limited success in recreating the complex and heterogeneous cellular organization found in vivo. More recently, new approaches based on developmental biology have focused on recreating morphogenesis underpinned by a more sophisticated molecular understanding, with the intent of harnessing the differentiation potential intrinsic in stem cells to allow for self-organization. Given the right cues, a number of increasingly complex structures have been recreated in vitro 371 (Fig. 2A), which for the first time may allow for "function-in-a-dish". 372 While their potential uses as replacement organs in regenerative medi- 373 cine is the clearest and ultimate objective, a more likely and tractable 374 shorter-term goal is to make use of such constructs in the context of 375 drug discovery.
376

A
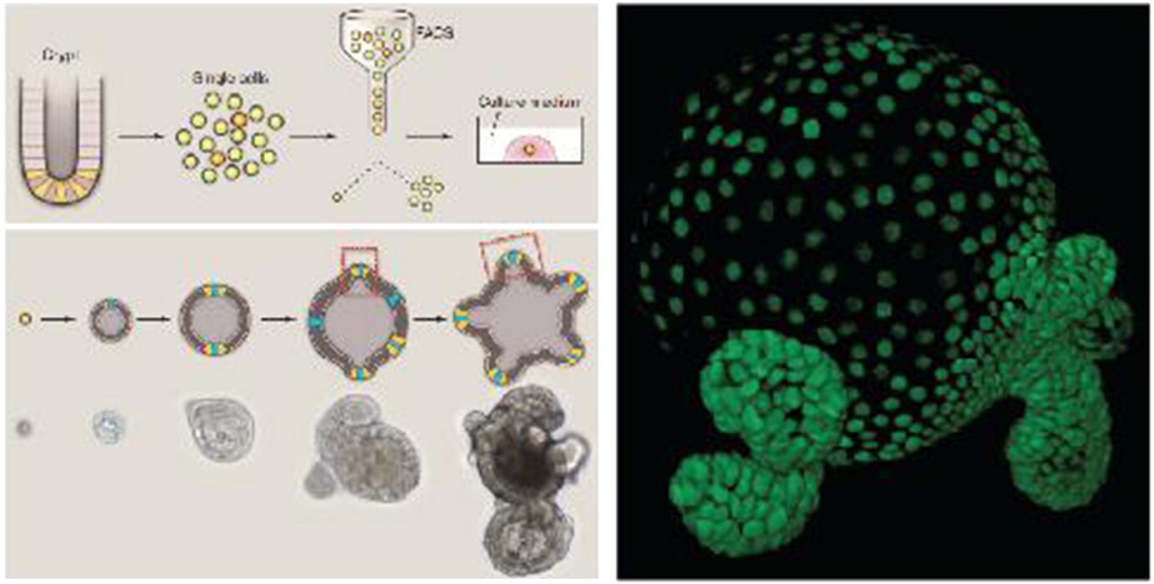

B
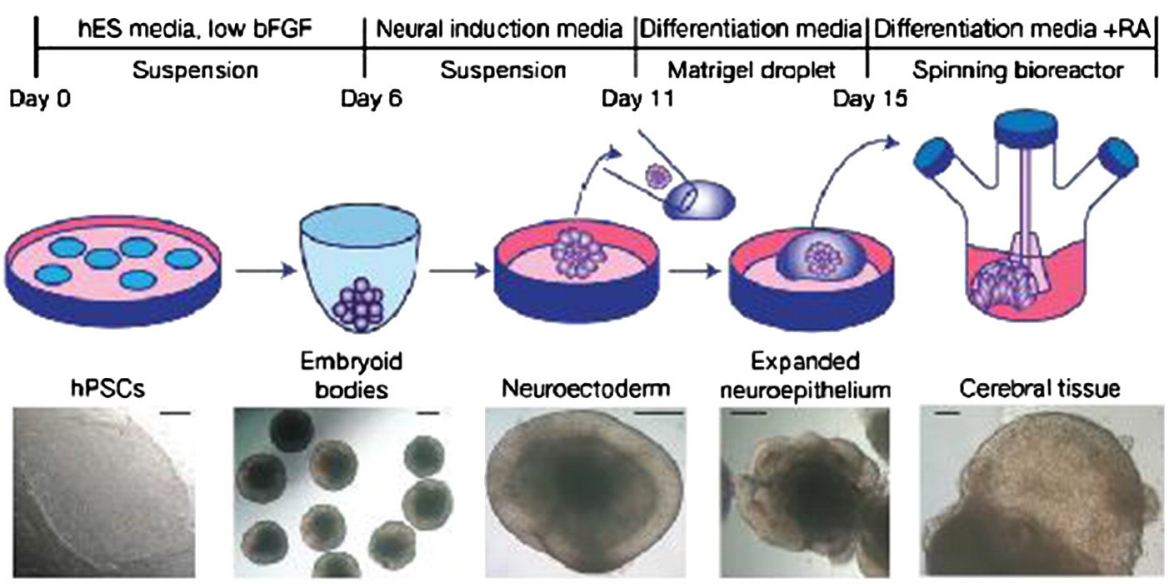

C

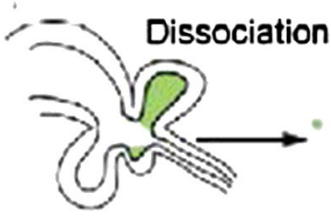

Day 1

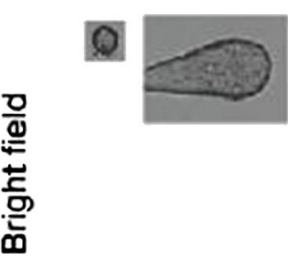

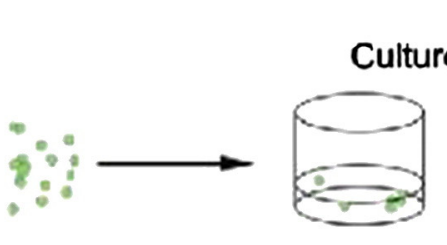

6

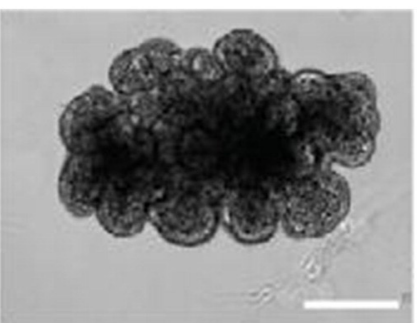

Culture in Matrigel ${ }^{\mathrm{T}}$ for 7 days

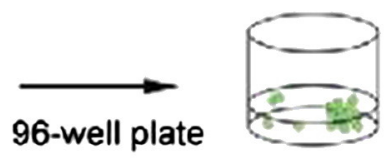

7

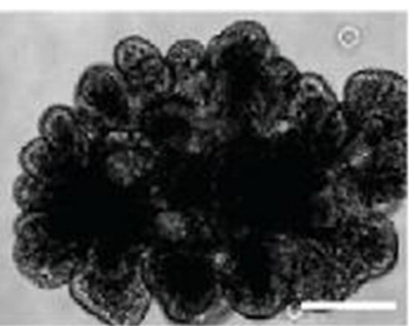

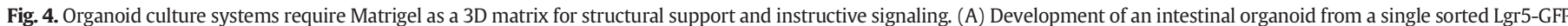

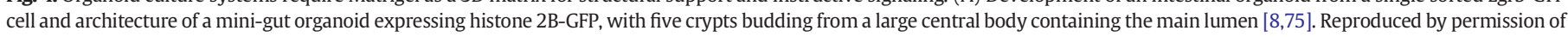

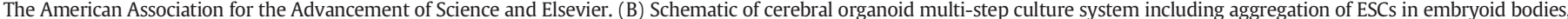

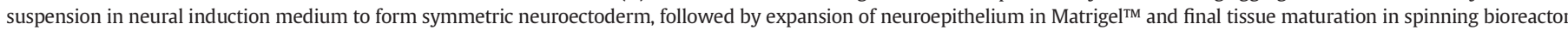

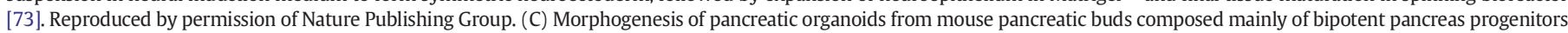
by seeding in growth factor-depleted Matrigel ${ }^{\mathrm{TM}}$ [53]. Branched organoid after 7 days contain 10,000 cells. Reproduced by permission of The Company of Biologists Limited. 
While spheroid-based approaches may be sufficient for testing drug efficacy in ablating tumor growth, mitigating migration and modeling to some extent angiogenesis, such approaches do not allow the equally important assessment of cytotoxic effects of drugs on a multitude of organs, nor the investigation of issues such as bioavailability when crossing the intestinal lining, liver metabolism, or blood-brain barrier effects. As key examples, we will focus here on recent developments in creating brain, liver and intestinal organoids (Fig. 2B), which could help in assessing these whole-organ effects.

\section{State-of-the-art organoid culture systems: Matrigel ${ }^{\mathrm{TM}}$ as critical component}

\subsection{Intestinal organoids}

In recent years, the organoid system that has gained the most attention is the mini-gut construct, or intestinal organoid (Fig. 4A). In landmark studies by the Clevers group [39-41], it was first shown that the transmembrane protein Lgr5 marks stem cells in the intestinal crypt, and that such cells exclusively contribute to the rapid self-renewal of the intestinal epithelium. Based on this in vivo knowledge, experiments were then carried out to demonstrate how an epithelial cell fragment isolated from the mouse intestinal crypt, when placed in an appropriate ex-vivo 3D culture system, could generate a 3D construct with some of the key characteristics of the intestine, including the establishment of a crypt-villi architecture as well as a lumenized interior [42]. Clearly, the stem cell niche concept plays a significant role in this system: factors such as R-Spondin, EGF, and Noggin are essential for the maintenance of the organoids in culture, and Matrigel ${ }^{\mathrm{TM}}$, the matrix used as 3D support, provides a set of structural and biochemical cues. Notably, it has been found that even a single Lgr5 cell could be sufficient to regrow an entire organoid, but this process occurred at very low efficiency (circa 5\%) [42]. Significantly, it has been shown that organoid-forming efficiency was greatly enhanced when Paneth cell-Lgr5 cell doublets were used, instead of single Lgr5 cells, suggesting that factors secreted by the Paneth support cell are crucial for regulating the intestinal niche [43]. Such intestinal organoids have also been derived from human ES cells [44], thereby greatly enhancing the applicability of such a system. In turn, it is now possible to envisage that such intestinal organoids could begin to be used to detect drug-intestine interactions, and, more specifically, to investigate bioavailability and aspects of drug pharmacokinetics. Indeed, intestinal organoids could certainly be imagined as complement or alternative to the commonly used Caco-2 monolayer transwell assay, which is now the norm as an in vitro model of human small intestinal mucosa's ability to absorb orally administered drugs [45].

\subsection{Cerebral organoids}

The developing human brain acquires its complexity through a myriad of developmental steps, with various cell types and regions acquiring their fate in a tightly regulated and sequential manner. In vitro, it has been possible to establish 2D cell culture protocols to generate bulk populations of neuronal subtypes from ESCs for screening purposes in procedures which have become fairly standardized and even in some cases deployed in primary drug screening assays [46]. With the adoption of 3D culture techniques, it has been possible in recent years to generate increasingly complex neural subsystems which, to some extent, preserve their highly restricted in vivo spatial arrangement. Indeed, the formation of an optic cup from ESCs, including a multilayered neural retina containing rods and cones, was shown to occur using a multi-step protocol involving the creation of aggregates in a floating culture in serum-free and growth-factor-reduced medium, named SFEBq culture, or serum-free culture of embryoid body-like aggregates with quick aggregation [47]. The extraordinary self-organization seen in the optic cup construct involves nonetheless, both in mouse and human systems
[48], the embedding of aggregates in Matrigel ${ }^{\mathrm{TM}}$. Indeed, while it may be 438 claimed that the process is entirely driven by spontaneous self- 439 organization orchestrated by local cellular interactions, it is quite possi- 440 ble that there are instructive matrix-derived cues at critical points in the 441 process, which may perhaps even be the initiating impetus for key 442 symmetry-breaking events within the homogeneous aggregates. More 443 recently, a similar SFEBq-based protocol has been utilized to develop a 444 human ESC-derived 3D organoid, termed cerebral organoids, where ex- 445 tensive patterning of brain regions can be seen, including characteristic 446 cerebral cortex zones with mature cortical neuron subtypes [49] 447 (Fig. 4B). In an elegant demonstration of how such an approach could 448 be used for disease modeling, such a cerebral organoid was modeled 449 from induced pluripotent stem (iPS) cells derived from a patient pre- 450 senting with microcephaly, a disorder which has yet to be suitably Q10 reproduced in a mouse model. Such organoids were less developed 452 than their normal counterparts, with an analysis of the constructs re- 453 vealing a potential mechanism for disease progression rooted in defec- 454 tive, premature neuronal differentiation. Proof-of-principle studies 455 such as this one confirm the promise that patient-derived iPS cells can 456 serve to better understand disease and to identify potential molecular 457 targets from a function perspective. In particular, cerebral organoids 458 could also be further developed to study the blood-brain barrier and 459 help overcome the difficulties in delivering pharmacological agents 460 into specific areas of the brain. Such an approach, which would require 461 at least some measure of vascularization, could potentially be achieved 462 within a co-culture system, and could potentially identify novel molec- 463 ular paths to entry or specific regions of the brain more sensitive to drug 464 delivery.

\subsection{Liver organoids}

Indeed, such a co-culture system has been employed in an approach 467 focused on generating a liver organoid. A liver bud exhibiting similar 468 markers to its in vivo counterpart was generated after aggregation of 469 three cell types at very high cell densities (human umbilical vein endo- 470 thelial cells (HUVEC), human mesenchymal stem cells (MSC) and iPS 471 cell-derived hepatic cells) and embedding in Matrigel ${ }^{\mathrm{TM}}$ [50]. As in the 472 neural and intestinal systems, self-organization occurred within the 473 organoids; here a notable advance involved the additional development 474 of nascent endothelial networks, which, helped by MSCs thought to 475 function as a source of pericytes promoting vessel stability [51], allowed 476 the limb bud to integrate into the host vasculature when implanted into 477 ectopic extrahepatic sites in a mouse. Within two months in vivo 478 these organoids matured and resembled adult liver histologically, and 479 had developed bile canaliculi (though not bile ducts). Importantly, 480 multiple transplanted liver organoids were able to rescue mice 481 from subacute gangyclovir-induced liver failure. As with other Q11 newly developed in vitro organoid systems, a fully mature miniatur- 483 ized organ with a complete set of functional features has not yet been 484 achieved, but it can be speculated that a better understanding of 485 complex signaling pathways involved in establishing morphogene- 486 sis, co-culture approaches and longer maturation times could lead 487 to the types of function seen here in this case after in vivo implanta- 488 tion. Still, even immature human-cell based organoids such as the 489 one depicted here, may be more responsive and predictive of acute 490 liver injury which is difficult to detect in the course of the current 491 drug discovery pipeline.

The strikingly complex organoid model systems described here rep- 493 resent only a selection from the rapidly expanding organoid literature. 494 Indeed, there have also been in the last year reports of such diverse 495 organoids as the pituitary gland [52], inner ear [49], pancreas [53,54] 496 (Fig. 4C) and hair follicle [55]. For all these systems, whether derived 497 from single cells or from pre-aggregated pluripotent stem cells, the 498 overarching feature has been a significant level of self-organization 499 over time mediated by a 3D matrix. 


\section{ECMs and 3D screening: towards synthetics and scalable approaches}

\subsection{Engineering better hydrogel systems}

A number of challenges present themselves in the pursuit of an effective translation of these organoid culture systems from an academic laboratory proof-of-concept to the kind of robust and reliable assay required for a drug discovery program. The first of these challenges is the need for a reproducible, well-defined and scalable 3D gel system. Indeed, all the organoid cultures presented in the previous section made use of the commercially available Matrige ${ }^{\mathrm{TM}}$ system, which is an extract from Engelbreth-Holm-Swarm (EHS) mouse sarcoma, a tumor rich in ECM proteins. Its main component is Laminin-1, an abundant extracellular component found in basement membrane, while other components of Matrigel ${ }^{\mathrm{TM}}$ include a mixture of collagen IV (30\%) and entactin (6\%) as well as heparin sulfate proteoglycans and a variety of growth factors in varying proportions, including transforming growth factor beta (TGFß), epidermal growth factor (EGF), fibroblast growth factor (FGF), tissue plasminogen activator, as well as residual matrix metalloproteinases (MMPs) and growth factors occurring naturally from in the tumor [56]. It appears clear that the organogenic bioactivity of this matrix therefore derives both not only from its three-dimensionality but also from the complex mixture of signaling cues. Yet, Matrigel ${ }^{\mathrm{TM}}$ remains a natural extract with relatively imprecise composition and unquantifiable batch-to-batch variability, rendering it unsuitable for reproducible and large-scale assays. Furthermore, Matrigel ${ }^{\mathrm{TM}}$ presents a number of practical limitations in handling and processing: it requires careful manipulation and must be maintained at constant cold temperature throughout cell-encapsulation processes, which is incompatible with current implementations of large-scale robotics, which have generally been adapted to handle cell culture reagents within a different temperature range. Matrigel ${ }^{\mathrm{TM}}$ also tends to have a widely variable degradation profile, which, depending on handling procedures as well as cell-mediated responses, can partially degrade in an uncontrolled manner within the time span of the assay.

Thus, to begin to consider the implementation of organoids as suitable in vitro drug discovery tools, a better-defined matrix which would minimize or preferably completely eliminate animal-derived components as well as be easy to handle and reproducibly degraded is necessary. While other purified natural materials such as alginate and collagen I have also been shown to support cell encapsulation, it is doubtful whether such materials could allow for the complex morphogenesis seen in Matrigel ${ }^{\mathrm{TM}}$, due to their relatively poor abilities to be functionalized with additional required signaling cues and lack of independent modulation of structural and chemical properties.

Currently, synthetic and highly tuneable approaches to materials engineering can provide hydrogels with the versatility and consistency which would be required for large-scale compound screening (reviewed in $[12,57]$ ). Such artificial ECMs can allow the experimenter to determine in an independent and highly reproducible manner both the physical and biochemical properties of such matrices. For example, poly(ethylene glycol) (PEG)-based hydrogel approaches allow us to create biophysically active materials. These materials allow for modulation of mechanical properties as well as cell-mediated degradation in response to matrix metalloprotease (MMP) secretion [13, 58,59]. For example, in a model of epithelial ovarian cancer, it was shown by exploiting the design flexibility of the hydrogel characteristics, that proliferation in 3D was dependent on cell-integrin engagement and on the ability of cells to proteolytically remodel their immediate extracellular microenvironment while maintain hydrogel stability in long-term culture [13]. Additionally, it is possible to chemically tether instructive ECM-derived signals directly onto the PEG backbone, thereby modulating the biochemical microenvironment. Indeed, by engineering protein or peptide constructs compatible with the chosen cross-linking system [60-62], it is possible to

build a material toolbox whose elements can potentially be used in 565 a set of combinatorial rearrangements [63].

While Matrigel ${ }^{\mathrm{TM}}$ presents multiple and potentially interacting cues, 568 defined synthetic matrices would need to be precisely tuned to achieve 569 the required optimal properties. In such a reductionist and defined ap- 570 proach, the faithful recreation of organoid microenvironments would 571 have to go beyond the "one-size-fits-all" approach. A Matrigel ${ }^{\mathrm{TM}}$-like 572 organ-specific synthetic analog would certainly require an as yet un- 573 known combination of biophysical properties and biochemical signaling 574 cues, and it would be necessary to establish a screening paradigm to sys- 575 tematically identify unique microenvironments which would optimally 576 support robust and reproducible organoid development. $\quad 577$

A number of approaches have been proposed to begin to assess the 578 effect of large-scale combinatorial biomaterial libraries on cell behavior. 579 The repurposing of DNA microarray printers to produce what have been 580 termed "cellular microarrays" has been a particularly popular tech- 581 nique. In one implementation, a combination of synthetic polymers 582 with different material properties including wettability, surface topog- 583 raphy, surface chemistry and elastic modulus were arrayed onto glass 584 slides and were assessed for their ability to maintain human ESC self- 585 renewal [64]. In another example, ECM proteins and soluble factors 586 were assessed in combination to determine optimal conditions for pri- 587 mary hepatocyte maintenance and early hepatic differentiation of 588 ESCs [65]. These platforms focused on directly functionalizing micro- 589 scope slides have relied on cell adhesion to provide a cellular readout. 590 Other approaches have focused on the creation of structured microwell 591 arrays which served to entrap cells and could for example track individ- 592 ual cell fate in a more precise manner via time-lapse microscopy, and 593 platforms where the simultaneous physical and biochemical properties 594 of the matrix (i.e. hydrogel substrate stiffness and surface protein 595

A
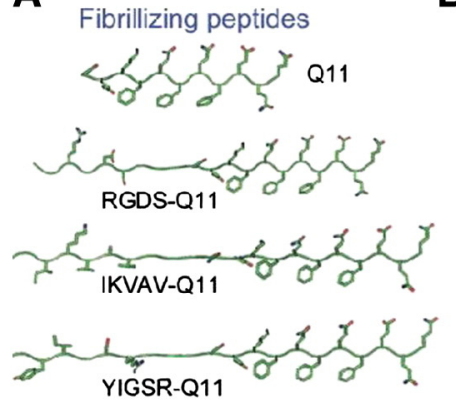

C

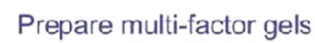
in multi-well plate

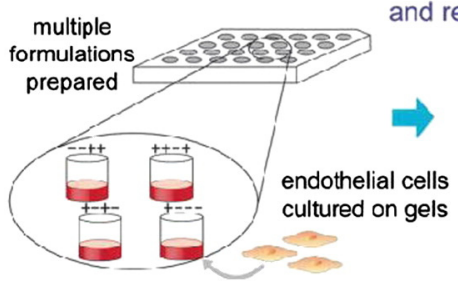

Optimize multiple factors using factorial experiments and response surface methodology

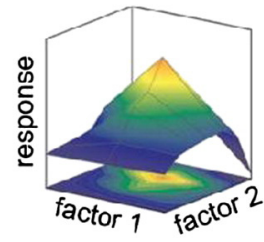

Fig. 5. Modular 3D scaffolds enable multifactorial experimentation [68]. Different ligandbearing peptides were synthesized (A) and co-assembled into fibrillar hydrogels (B). HUVECs were cultured in combination of gels with different combinations of peptides (C). Full factorial experiments and response surface methodology were used to explore the direct and interactive effects of various immobilized ligands on cell growth, and optimum formulations that maximized proliferation were identified (D). Reproduced by permission of RSC Publishing. 
functionalization) that could be modulated have enabled truly multifactorial explorations of extrinsic microenvironmental control [66].

\subsection{Challenges in implementing engineered organoids}

While much can be learned from the combinatorial technologies seen above, a major limitation has remained that only adherent cells or cellular aggregates such as neurospheres [67] in liquid media could be assayed in such systems. Despite technical difficulties which have hampered the use of high-throughput combinatorial studies in 3D, the deployment of rational approaches based on design of experiment (DOE) methodologies has proved to be instructive in ways to assess a combinatorial space for 3D cellular response. In one notable example, multiple peptide ligands were incorporated into engineered selfassembling peptide hydrogels. An iterative process consisting of single-factor experiments for setting initial bounds followed by factorial experiments for identifying main effects and interactions between ligands served to identify previously unknown antagonistic interaction between the laminin-derived peptide mediating HUVEC cell attachment and growth. In a final step, response surface methodology experiments were carried out to identify optimal formulations of these ligands, which led to endothelial cell growth equivalent to that on native full-length fibronectin [68] (Fig. 5).

Ultimately, once such synthetic matrices would be defined for an organoid of interest, it is possible that such artificial extracellular microenvironments could be widely deployed, either in standardized formats using liquid handling robots, or via miniaturized arrays such as the ones described for toxicity testing [69]. With organoid morphology and function being significantly more complex than spheroids or single cells, one important challenge will be in the systematic assessment of function. More advanced readouts would have to be developed to address these issues; while confocal microscopy currently provides the standard imaging tool for assessing cellular function within such constructs, it is certainly limited in throughput. As such, newer techniques currently being developed for whole animal imaging allowing for rapid and highly accurate scanning of large areas, such as light sheet fluorescence microscopy or high-resolution optical coherence tomography could be deployed in this context. Light-sheet microscopy, where only the fluorophores in the light sheet's plane contribute to the image, is particularly useful in reducing out-of-focus blur from three-dimensional samples [70]. Additionally, a technique known as biodynamic imaging, which uses shortcoherence dynamic light scattering to evaluate intracellular motions [71], has recently been specifically applied to study the multicellular cancer spheroid model. However, the key for such promising new imaging technologies to gain wider application will be to modify them in appropriate ways in order to conform to the standardized formats and throughput required in the drug discovery process. Furthermore, image analysis tools focused on simple cellular morphologies would have to be adapted and customized to yield relevant and quantitative data. Beyond imaging, a number of multiplexed techniques could also be used to interrogate for functional outputs and to maximize sample value. For example, Luminex/XMAP bead-based assays can be used to detect hundreds of proteins or genes of interest within a single sample, while gene expression can be assessed by such technologies as the Fluidigm qPCR gene expression profiling system [72] as well with as a battery of increasingly inexpensive sequencing technologies. Today, some pharmaceutical companies are actively.

\section{Conclusions and outlook}

Numerous studies have now shown the advantages of 3D cell culture, in particular in the context of the multi-cellular tumor spheroid model, with notably different drug responses compared to 2D contexts which in some cases compare favorably to the in vivo observations. Such 3D cultures have also been used to demonstrate more physiological responses in other contexts such as migration, invasion, angiogenesis and lymphangiogenesis, as well as in toxicology. We have proposed in this 658 review that complex self-organized organoids, which have recently 659 come to the fore as striking proof-of-concept examples of in vitro devel- 660 opmental biology, could be appropriate test platforms for future drug 661 discovery efforts. Indeed, such miniaturized proto-organs could be used 662 as a significant validation bridge between primary high-throughput 663 screening and costly animal and human trials. Safety and efficacy of 664 lead compounds could be tested directly on in vitro organs for the target 665 pathology. It could also be imagined that in the context of oncology, cells 666 and tumor response could be assessed not only in isolation but in a more 667 realistic co-culture system within an organ of interest. Ultimately, such 668 organoid systems could be systemically linked in order to begin to 669 build a "human in a dish" as a technology with unprecedented fidelity 670 to human disease and drug response. Today, the promise of such trans- 671 formative advances is largely limited by issues of historical importance 672 in the drug discovery process: reproducibility, standardization, valida- 673 tion and quality control. For these far-reaching objectives to be accom- 674 plished and for such technologies to move from the university 675 laboratory to a broader use in the commercial drug discovery process, 676 we have emphasized the need to focus on implementations based on 677 synthetic and tailored 3D matrices amenable to medium to large-scale 678 automation, as well as meaningful multiplexed readouts.

\section{References}

[1] C.P. Adams, V.V. Brantner, Estimating the cost of new drug development: is it really 681 802 million dollars? Health Aff. (Millwood) 25 (2006) 420-428.

[2] K. Tsaioun, M. Jacewicz, De-risking drug discovery with ADDME - avoiding drug 683 development mistakes early, Altern. Lab. Anim. 37 (Suppl. 1) (2009) 47-55. 684

[3] K. Tsaioun, M. Bottlaender, A. Mabondzo, ADDME-Avoiding Drug Development 685 Mistakes Early: central nervous system drug discovery perspective, BMC Neurol. 9686 (Suppl. 1) (2009) S1.

[4] J.A. DiMasi, R.W. Hansen, H.G. Grabowski, The price of innovation: new estimates of 688 drug development costs, J. Health Econ. 22 (2003) 151-185.

[5] D.V. LaBarbera, B.G. Reid, B.H. Yoo, The multicellular tumor spheroid model for 690 high-throughput cancer drug discovery, Expert Opin. Drug Discovery 7 (2012) 691 819-830.

[6] L.A. Kunz-Schughart, J.P. Freyer, F. Hofstaedter, R. Ebner, The use of 3-D cultures for 693 high-throughput screening: the multicellular spheroid model, J. Biomol. Screen. 9694 (2004) 273-285.

[7] A.M. Ghaemmaghami, M.J. Hancock, H. Harrington, H. Kaji, A. Khademhosseini, Bio- 696 mimetic tissues on a chip for drug discovery, Drug Discov. Today 17 (2012) 697 173-181.

[8] H. Clevers. The intestinal crypt a prototype stem cell compartment Cell 154 (2013) 699 274-284.

[9] Y. Sasai, M. Eiraku, H. Suga, In vitro organogenesis in three dimensions: 701 self-organising stem cells, Development 139 (2012) 4111-4121:

[10] Y. Sasai, Next-generation regenerative medicine: organogenesis from stem cells in 703 3D culture, Cell Stem Cell 12 (2013) 520-530.

[11] D.C. Swinney, J. Anthony, How were new medicines discovered? Nat. Rev. Drug 705 Discov. 10 (2011) 507-519.

[12] M. Rimann, U. Graf-Hausner, Synthetic 3D multicellular systems for drug develop- 707 ment, Curr. Opin. Biotechnol. 23 (2012) 803-809.

[13] D. Loessner, K.S. Stok, M.P. Lutolf, D.W. Hutmacher, J.A. Clements, S.C. Rizzi, 709 Bioengineered 3D platform to explore cell-ECM interactions and drug resistance 710 of epithelial ovarian cancer cells, Biomaterials 31 (2010) 8494-8506.

14] F. Hirschhaeuser, H. Menne, C. Dittfeld, J. West, W. Mueller-Klieser, L.A. 712 Kunz-Schughart, Multicellular tumor spheroids: an underestimated tool is catching 713 up again, J. Biotechnol. 148 (2010) 3-15.

[15] J. Friedrich, C. Seidel, R. Ebner, L.A. Kunz-Schughart, Spheroid-based drug screen: 715 considerations and practical approach, Nat. Protoc. 4 (2009) 309-324.

[16] A.Y. Hsiao, Y.C. Tung, C.H. Kuo, B. Mosadegh, R. Bedenis, K.J. Pienta, S. Takayama, 717 Micro-ring structures stabilize microdroplets to enable long term spheroid culture 718 in 384 hanging drop array plates, Biomed. Microdevices 14 (2012) 313-323. 719

[17] A.Y. Hsiao, Y.C. Tung, X.G. Qu, L.R. Patel, K.J. Pienta, S. Takayama, 384 hanging drop 720 arrays give excellent $Z$-factors and allow versatile formation of co-culture spheroids, 721 Biotechnol. Bioeng. 109 (2012) 1293-1304.

[18] Y.C. Tung, A.Y. Hsiao, S.G. Allen, Y.S. Torisawa, M. Ho, S. Takayama, High-throughput 723 3D spheroid culture and drug testing using a 384 hanging drop array, Analyst 136724 (2011) 473-478.

[19] C.R. Thoma, S. Stroebel, N. Rosch, B. Calpe, W. Krek, J.M. Kelm, A high-throughput- 726 compatible 3D microtissue co-culture system for phenotypic RNAi screening appli- 727 cations, J. Biomol. Screen. 18 (2013) 1330-1337.

[20] E. Ueda, F.L. Geyer, V. Nedashkivska, P.A. Levkin, DropletMicroarray: facile formation 729 of arrays of microdroplets and hydrogel micropads for cell screening applications, 730 Lab Chip 12 (2012) 5218-5224.

[21] V.H. Ho, W.M. Guo, C.L. Huang, S.F. Ho, S.Y. Chaw, E.Y. Tan, K.W. Ng, J.S. Loo, Manip- 732 ulating magnetic 3D spheroids in hanging drops for applications in tissue engineer- 733 ing and drug screening, Adv. Healthc. Mater. (2013). 
[22] P. Longati, X. Jia, J. Eimer, A. Wagman, M.R. Witt, S. Rehnmark, C. Verbeke, R. Toftgard, M. Lohr, R.L. Heuchel, 3D pancreatic carcinoma spheroids induce a matrix-rich, chemoresistant phenotype offering a better model for drug testing, BMC Cancer 13 (2013) 95

[23] S.R. Horman, J. To, A.P. Orth, An HTS-compatible 3D colony formation assay to identify tumor-specific chemotherapeutics, J. Biomol. Screen. (2013).

[24] C. Godugu, A.R. Patel, U. Desai, T. Andey, A. Sams, M. Singh, AlgiMatrix (TM) based 3D cell culture system as an in-vitro tumor model for anticancer studies, PLoS One 8 (2013).

[25] M. Zimmermann, C. Box, S.A. Eccles, Two-dimensional vs. three-dimensional in vitro tumor migration and invasion assays, Methods Mol. Biol. 986 (2013) 227-252.

[26] D.H. Nguyen, S.C. Stapleton, M.T. Yang, S.S. Cha, C.K. Choi, P.A. Galie, C.S. Chen, Biomimetic model to reconstitute angiogenic sprouting morphogenesis in vitro, Proc. Natl. Acad. Sci. U. S. A. 110 (2013) 6712-6717.

[27] C. Fischbach, H.J. Kong, S.X. Hsiong, M.B. Evangelista, W. Yuen, D.J. Mooney, Cancer cell angiogenic capability is regulated by $3 \mathrm{D}$ culture and integrin engagement, Proc. Natl. Acad. Sci. U. S. A. 106 (2009) 399-404.

[28] W.A. Farahat, L.B. Wood, I.K. Zervantonakis, A. Schor, S. Ong, D. Neal, R.D. Kamm, H.H. Asada, Ensemble analysis of angiogenic growth in three-dimensional microfluidic cell cultures, PLoS One 7 (2012) 25.

[29] G. Seano, G. Chiaverina, P.A. Gagliardi, L. di Blasio, R. Sessa, F. Bussolino, L. Primo, Modeling human tumor angiogenesis in a three-dimensional culture system, Blood 121 (2013) (2012-2008).

[30] M.M. Schulz, F. Reisen, S. Zgraggen, S. Fischer, D. Yuen, G.J. Kang, L. Chen, G. Schneider, M. Detmar, Phenotype-based high-content chemical library screening identifies statins as inhibitors of in vivo lymphangiogenesis, Proc. Natl. Acad. Sci. U. S. A. 109 (2012) E2665-E2674.

[31] D. Mueller, L. Kramer, E. Hoffmann, S. Klein, F. Noor, 3D organotypic HepaRC cultures as in vitro model for acute and repeated dose toxicity studies, Toxicol. in Vitro 9 (2013) 00176-00178.

[32] P. Gunness, D. Mueller, V. Shevchenko, E. Heinzle, M. Ingelman-Sundberg, F. Noor, 3D organotypic cultures of human HepaRG cells: a tool for in vitro toxicity studies, Toxicol. Sci. 133 (2013) 67-78.

[33] S. Messner, I. Agarkova, W. Moritz, J.M. Kelm, Multi-cell type human liver microtissues for hepatotoxicity testing, Arch. Toxicol. 87 (2013) 209-213.

[34] R. Kostadinova, F. Boess, D. Applegate, L. Suter, T. Weiser, T. Singer, B. Naughton, A. Roth, A long-term three dimensional liver co-culture system for improved prediction of clinically relevant drug-induced hepatotoxicity, Toxicol. Appl. Pharmacol. 268 (2013) 1-16

[35] H. Olson, G. Betton, D. Robinson, K. Thomas, A. Monro, G. Kolaja, P. Lilly, J. Sanders, G Sipes, W. Bracken, M. Dorato, K. Van Deun, P. Smith, B. Berger, A. Heller, Concordance of the toxicity of pharmaceuticals in humans and in animals, Regul. Toxicol Pharmacol. 32 (2000) 56-67.

[36] J. Seok, H.S. Warren, A.G. Cuenca, M.N. Mindrinos, H.V. Baker, W. Xu, D.R. Richards, G.P. McDonald-Smith, H. Gao, L. Hennessy, C.C. Finnerty, C.M. Lopez, S. Honari, E.E. Moore, J.P. Minei, J. Cuschieri, P.E. Bankey, J.L. Johnson, J. Sperry, A.B. Nathens, T.R. Billiar, M.A. West, M.G. Jeschke, M.B. Klein, R.L Gamelli, N.S. Gibran, B.H. Brownstein, C. Miller-Graziano, S.E. Calvano, P.H. Mason, J.P. Cobb, L.G. Rahme, S.F. Lowry, R.V. Maier, L.L. Moldawer, D.N. Herndon, R.W. Davis, W. Xiao, R.G. Tompkins, Genomic responses in mouse models poorly mimic human inflammatory diseases, Proc. Natl. Acad. Sci. U. S. A. 110 (2013) 3507-3512.

[37] M. Huch, S.F. Boj, H. Clevers, Lgr5(+) liver stem cells, hepatic organoids and regenerative medicine, Regen. Med. 8 (2013) 385-387.

[38] D. Solter, From teratocarcinomas to embryonic stem cells and beyond: a history of embryonic stem cell research, Nat. Rev. Genet. 7 (2006) 319-327.

[39] N. Barker, J.H. van Es, J. Kuipers, P. Kujala, M. van den Born, M. Cozijnsen, A. Haegebarth, J. Korving, H. Begthel, P.J. Peters, H. Clevers, Identification of stem cells in small intestine and colon by marker gene Lgr5, Nature 449 (2007) 1003-1007.

[40] N. Barker, R.A. Ridgway, J.H. van Es, M. van de Wetering, H. Begthel, M. van den Born, E. Danenberg A.R. Clarke, O.J. Sansom, H. Clevers, Crypt stem cells as the cells-of-origin of intestinal cancer, Nature 457 (2009) 608-U119.

[41] H.J. Snippert, L.G. van der Flier, T. Sato, J.H. van Es, M. van den Born, C. Kroon-Veenboer, N. Barker, A.M. Klein, J. van Rheenen, B.D. Simons, H. Clevers, Intestinal crypt homeostasis results from neutral competition between symmetrically dividing Lgr5 stem cells, Cell 143 (2010) 134-144.

[42] T. Sato, R.G. Vries, H.J. Snippert, M. van de Wetering, N. Barker, D.E. Stange, J.H. van Es, A. Abo, P. Kujala, P.J. Peters, H. Clevers, Single Lgr5 stem cells build crypt-villus structures in vitro without a mesenchymal niche, Nature 459 (2009) 262-U147.

[43] T. Sato, J.H. van Es, H.J. Snippert, D.E. Stange, R.G. Vries, M. van den Born, N. Barker N.F. Shroyer, M. van de Wetering, H. Clevers, Paneth cells constitute the niche for Lgr5 stem cells in intestinal crypts, Nature 469 (2011) 415-418.

[44] J.R. Spence, C.N. Mayhew, S.A. Rankin, M.F. Kuhar, J.E. Vallance, K. Tolle, E.E. Hoskins, V.V. Kalinichenko, S.I. Wells, A.M. Zorn, N.F. Shroyer, J.M. Wells, Directed differentiation of human pluripotent stem cells into intestinal tissue in vitro, Nature 470 (2011) 105-U120.

[45] L. Fossati, R. Dechaume, E. Hardillier, D. Chevillon, C. Prevost, S. Bolze, N. Maubon, Use of simulated intestinal fluid for Caco-2 permeability assay of lipophilic drugs, Int. J. Pharm. 360 (2008) 148-155.

[46] A. Efthymiou, A. Shaltouki, J.P. Steiner, B. Jha, S.M. Heman-Ackah, A. Swistowski, X. Zeng, M.S. Rao, N. Malik, Functional screening assays with neurons generated from pluripotent stem cell-derived neural stem cells, J. Biomol. Screen. (2013).

[47] M. Eiraku, Y. Sasai, Self-organizing optic-cup morphogenesis in three-dimensional culture, Neurosci. Res. 71 (2011) E127-E128.
[48] T. Nakano, S. Ando, N. Takata, M. Kawada, K. Muguruma, K. Sekiguchi, K. Saito, S. 819 Yonemura, M. Eiraku, Y. Sasai, Self-formation of optic cups and storable stratified 820 neural retina from human ESCs, Cell Stem Cell 10 (2012) 771-785.

[49] K.R. Koehler, A.M. Mikosz, A.I. Molosh, D. Patel, E. Hashino, Generation of inner ear 822 sensory epithelia from pluripotent stem cells in 3D culture, Nature 500 (2013) 823 $217-221$.

[50] T. Takebe, K. Sekine, M. Enomura, H. Koike, M. Kimura, T. Ogaeri, R.R. Zhang, Y. Ueno, 825 Y.W. Zheng, N. Koike, S. Aoyama, Y. Adachi, H. Taniguchi, Vascularized and function- 826 al human liver from an iPSC-derived organ bud transplant, Nature 499 (2013) 827 $481-484$.

[51] H. Willenbring, A. Soto-Gutierrez, Transplantable liver organoids made from only 829 three ingredients, Cell Stem Cell 13 (2013) 139-140.

[52] H. Suga, T. Kadoshima, M. Minaguchi, M. Ohgushi, M. Soen, T. Nakano, N. Takata, T. Q2 Wataya, K. Muguruma, H. Miyoshi, S. Yonemura, Y. Oiso, Y. Sasai, Self-formation of 832 functional adenohypophysis in three-dimensional culture, Nature 480 (2011) 833 57-U215.

[53] C. Greggio, F. De Franceschi, M. Figueiredo-Larsen, S. Gobaa, A. Ranga, H. Semb, M. 835 Lutolf, A. Grapin-Botton, Artificial three-dimensional niches deconstruct pancreas 836 development in vitro, Development 140 (2013) 4452-4462.

[54] M. Huch, P. Bonfanti, S.F. Boj, T. Sato, C.J. Loomans, M. van de Wetering, M. Sojoodi, 838 V.S. Li, J. Schuijers, A. Gracanin, F. Ringnalda, H. Begthel, K. Hamer, J. Mulder, J.H. van 839 Es, E. de Koning. R.G. Vries, H. Heimberg. H. Clevers, Unlimited in vitro expansion of 840 adult bi-potent pancreas progenitors through the Lgr5/R-spondin axis, EMBO J. 32841 (2013) 2708-2721.

[55] C.A. Higgins, J.C. Chen, J.E. Cerise, C.A. Jahoda, A.M. Christiano, Microenvironmental 843 reprogramming by three-dimensional culture enables dermal papilla cells to induce 844 de novo human hair-follicle growth, Proc. Natl. Acad. Sci. U. S. A. 21 (2013) $21 . \quad 845$

[56] H.K. Kleinman, G.R. Martin, Matrigel: basement membrane matrix with biological 846 activity, Semin. Cancer Biol. 15 (2005) 378-386.

[57] M.P. Lutolf, P.M. Gilbert, H.M. Blau, Designing materials to direct stem-cell fate, 848 Nature 462 (2009) 433-441.

[58] M. Ehrbar, S.C. Rizzi, R.G. Schoenmakers, B.S. Miguel, J.A. Hubbell, F.E. Weber, M.P. 850 Lutolf, Biomolecular hydrogels formed and degraded via site-specific enzymatic 851 reactions, Biomacromolecules 8 (2007) 3000-3007.

[59] M.P. Lutolf, J.A. Hubbell, Synthetic biomaterials as instructive extracellular microen- 853 vironments for morphogenesis in tissue engineering, Nat. Biotechnol. 23 (2005) 854 47-55.

[60] F. Tortelli, M. Pisano, P.S. Briquez, M.M. Martino, J.A. Hubbell, Fibronectin binding 856 modulates CXCL11 activity and facilitates wound healing, PLoS One 8 (2013) 857 0079610.

[61] M.M. Martino, P.S. Briquez, A. Ranga, M.P. Lutolf, J.A. Hubbell, Heparin-binding do- 859 main of fibrin(ogen) binds growth factors and promotes tissue repair when incor- 860 porated within a synthetic matrix, Proc. Natl. Acad. Sci. U. S. A. 110 (2013) 861 4563-4568.

[62] M.M. Martino, F. Tortelli, M. Mochizuki, S. Traub, D. Ben-David, G.A. Kuhn, R. Muller, 863 E. Livne, S.A. Eming, J.A. Hubbell, Engineering the growth factor microenvironment 864 with fibronectin domains to promote wound and bone tissue healing Sci. Transl. 865 Med. 3 (2011) 3002614

[63] A. Ranga, M.P. Lutolf, High-throughput approaches for the analysis of extrinsic 867 regulators of stem cell fate, Curr. Opin. Cell Biol. 24 (2012) 236-244.

[64] Y. Mei, K. Saha, S.R. Bogatyrev, J. Yang, A.L. Hook, Z.I. Kalcioglu, S.W. Cho, M. 869 Mitalipova, N. Pyzocha, F. Rojas, K.J. Van Vliet, M.C. Davies, M.R. Alexander, R. 870 Langer, R. Jaenisch, D.G. Anderson, Combinatorial development of biomaterials for 871 clonal growth of human pluripotent stem cells, Nat. Mater. 9 (2010) 768-778. 872

[65] C.J. Flaim, S. Chien, S.N. Bhatia, An extracellular matrix microarray for probing cellu- 873 lar differentiation, Nat. Methods 2 (2005) 119-125.

[66] S. Gobaa, S. Hoehnel, M. Roccio, A. Negro, S. Kobel, M.P. Lutolf, Artificial niche micro- 875 arrays for probing single stem cell fate in high throughput, Nat. Methods 8 (2011) 876 949-955.

67] M. Cordey, M. Limacher, S. Kobel, V. Taylor, M.P. Lutolf, Enhancing the reliability and 878 throughput of neurosphere culture on hydrogel microwell arrays, Stem Cells 26879 (2008) 2586-2594.

[68] J.P. Jung, J.V. Moyano, J.H. Collier, Multifactorial optimization of endothelial cell 881 growth using modular synthetic extracellular matrices, Integr. Biol. 3 (2011) 882 185-196.

[69] L. Meli, E.T. Jordan, D.S. Clark, R.J. Linhardt, J.S. Dordick, Influence of a three- 884 dimensional, microarray environment on human cell culture in drug screening 885 systems, Biomaterials 33 (2012) 9087-9096.

[70] J. Swoger, F. Pampaloni, E.H. Stelzer, Cold Spring Harb. Protoc. 1 (2014). 887

[71] R. An, D. Merrill, L. Avramova, J. Sturgis, M. Tsiper, J.P. Robinson, J. Turek, D.D. Nolte, 888 Phenotypic profiling of Raf inhibitors and mitochondrial toxicity in 3D tissue using 889 biodynamic imaging, J. Biomol. Screen. 20 (2013) 20.

[72] Y. Zhou, T. Arai, Y. Horiguchi, K. Ino, T. Matsue, H. Shiku, Multiparameter analyses of 891 three-dimensionally cultured tumor spheroids based on respiratory activity and 892 comprehensive gene expression profiles, Anal. Biochem. 439 (2013) 187-193. 893

[73] M.A. Lancaster, M. Renner, C.A. Martin, D. Wenzel, L.S. Bicknell, M.E. Hurles, T. 894 Homfray, J.M. Penninger, A.P. Jackson, J.A. Knoblich, Cerebral organoids model 895 human brain development and microcephaly, Nature 501 (2013) 373-379. 896

[74] G. Seano, G. Chiaverina, P.A. Gagliardi, L. di Blasio, R. Sessa, F. Bussolino, L. Primo, 897 Modeling human tumor angiogenesis in a three-dimensional culture system, 898 Blood 121 (2013) e129-e137.

[75] T. Sato, H. Clevers, Growing self-organizing mini-guts from a single intestinal stem 900 cell: mechanism and applications, Science 340 (2013) 1190-1194.
901 